\title{
The increased risk of venous thromboembolism by advancing age cannot be attributed to the higher incidence of cancer in the elderly: the Tromsø study
}

Kristine Blix, ${ }^{1}$ Sigrid K. Brækkan, ${ }^{1,2}$ Saskia le Cessie, ${ }^{3}$ Finn E. Skjeldestad, ${ }^{4}$ Suzanne C. Cannegieter, ${ }^{3}$ John-

Bjarne Hansen. ${ }^{1,2}$

${ }^{1}$ Hematological Research Group, Department of Clinical Medicine, University of Tromsø, Norway

${ }^{2}$ Section for Hematology, Division of Internal Medicine, University Hospital of North Norway

${ }^{3}$ Department of Clinical Epidemiology, Leiden University Medical Center, the Netherlands

${ }^{4}$ Women's Health and Perinatology Research Group, Department of Clinical Medicine, University of Tromsø,

Norway

Running head: Blix et al. Cancer and VTE

Word count abstract: 200

Word count text: 4087

Number of tables: 5

Number of figures: 1

Correspondence to: Kristine Blix, Hematological Research Group, Department of Clinical Medicine, University of Tromsø, 9037 Tromsø, Norway.

Phone: + 47 77620994, Fax: +47 77646838, e-mail: Kristine.blix@uit.no 


\section{Abstract}

Whether the high incidence of venous thromboembolism (VTE) in the elderly can be attributed to cancer is not wellstudied. We assessed the impact of cancer on risk of VTE in young, middle-aged and elderly. 26,094 subjects without a history of cancer or VTE were recruited from the Tromsø study. Incident cancer $(n=2,290)$ and VTE (n=531) were recorded from baseline (1994-95) through December 31st, 2009. Cox regression with cancer as time-varying exposure was used to calculate hazard ratios with 95\% confidence intervals (CI). Overt cancer was associated with a 5-fold (95\%CI: 4.3, 6.7) increased risk of VTE, with an age-dependent gradient from 26 -fold (95 \%CI: 12.1, 56.5) increased in the young, 9-fold (95\% CI: 6.6, 12.7) increased in the middleaged, and 3-fold (95 \% CI: 2.5, 4.5) increased risk in the elderly. The population attributable risks were 14\%, $27 \%$ and $18 \%$, respectively. Conclusion: The relative risk of VTE by cancer were higher in young compared to elderly subjects, but the proportion of VTEs in the population due to cancer did not differ much across age groups. Our findings indicate that the increased risk of VTE by advancing age cannot be attributed to higher incidence of cancer in the elderly.

Keywords: venous thromboembolism, cancer, agegroups, cohort study, attributable risk 


\section{Introduction}

Deep vein thrombosis (DVT) and pulmonary embolism (PE), together referred to as venous thromboembolism (VTE), are frequent and life threatening complications of malignancy[1, 2].An increased incidence of VTE in patients with cancer has been unambiguously demonstrated[3], and the risk is shown to be particularly increased during the first few months after diagnosis as well as in the presence of distant metastases[4]. Overall, cancer is responsible for up to $25 \%$ of all incident VTE cases[2, 5, 6].The aetiology of cancer associated VTE is heterogeneous, and pathogenic mechanisms include tumour induced hypercoagulability, vascular injury caused by the tumour, chemotherapy or surgery, long term central venous catheters, venous stasis among bedridden patients, and tumour-dependent compression of the venous circulation[7].In subjects with cancer, a VTE event is associated with more frequent and prolonged hospital stays[8],more severe treatment complications[9],increased risk of recurrence[9, 10] and shortened life-expectancy compared to cancer patients without VTE[11, 12].

The incidence of VTE is strongly age-dependent, and varies from 1 per 100000 per year in young subjects to $1 \%$ per year in the elderly[2, 13].Likewise, malignant disease is substantially more prevalent at higher ages[14]. Thus, it is reasonable to assume that cancer contributes to the increased VTE incidence observed in the elderly population. However, despite the well-known association between cancer and VTE, limited data exist on the strength of the associationat different ages. A few studies have indicated that cancer may be less of a risk factor in old age than at younger ages[15, 16].In a small sub-study of the Multiple Environmental and Genetic Assessment of risk factors for venous thrombosis (MEGA) study, the relative risk of VTE was substantially higherin those younger than 70 compared to the elderly[15].Moreover, a Danish population-based registry study with hospitalization for VTE as primary outcome,showed that the relative risk of cancer associated VTEwas highest in the younger age groups[16].Similar findings were recently reported in a study using United Kingdomlinked databases[17].

Several statistical measures can be used to express the importanceof a causal risk factor in development of disease, and both the frequency of the exposure and the strength of the association need to be considered.The attributable risk (AR\%) represents the share of events among the exposed that can be explained by the exposure, whereas the population attributable risks (PAR\%) represent the share of events in a population that can be explained by a specific exposure.

The increased risk of VTE observed in the elderly is not well understood. Therefore, the aim of the present study was to investigate whether the increased risk of VTE at high age could be attributed to cancer.We 
used a general population cohort to assess future age-specificincidence rates and relative risksof VTE in patients withoccult and overt cancer. AR\% and PAR\% were calculated to illustrate the impact of cancer on venous thromboembolism in the young, middle-aged and elderly.

\section{Methods}

Study population and baseline measurements

Participants were recruited from the fourth survey of the Tromsø study (conducted in 1994-95), a single-centre, population-based cohort study. All inhabitants of Tromsø municipality $>24$ years were invited and 27,157 (77\% of the eligible population) participated. The study was approved by the regional committee of research ethics, and all participants gave their informed written consent to participate. Subjects who did not approve of medical research ( $\mathrm{n}=201)$ and subjects no longer registered as inhabitants of Tromsø municipality ( $\mathrm{n}=44)$, were excluded from the study. Furthermore, subjects with a known medical history of VTE (n=53)or history of cancer(n=763) were excluded. Thus, the baseline population included 26094 subjects with no priorcancer or VTE.

Baseline information was obtained by physical examination and self-administered questionnaires. Body height and weight were measured with the participants wearing light clothing and no shoes. The body mass index (BMI) was calculated as the weight in kilograms divided by the square of the height in meters $\left(\mathrm{kg} / \mathrm{m}^{2}\right)$. Information about physical activity, smoking habits, diabetes and previous cardiovascular diseases (angina, myocardial infarction and stroke) were obtained from self-administered questionnaires. The questions for physical activity were "How many times per week do you usually perform light or hard physical activity" (none, less than once per week, one-two times per week or three or more times per week for the two respective questions). The questions used to assess smoking status were "Do you smoke cigarettes, cigars or pipe daily" (yes or no for each tobacco).

\section{Cancer assessment}

Information on incident cancer during follow-up, e.g. date of cancer diagnosis and location of malignancy (International Classification of Disease, Revision 7 (ICD-7) codes 140-205), excluding non-melanoma skin cancer(ICD-7 191.0-191.9), was obtained from the Cancer Registry of Norway. In a recent evaluation of the data quality, completeness of reporting was estimated to $98.8 \%$, whereas organ specific morphology had $94 \%$ accuracy[18]. 
Venous thromboembolism assessment

Potential first life time diagnoses of VTE were identified by searching the hospital discharge diagnosis registry, the autopsy registry and the radiology registry at the University Hospital of North Norway, as previously described[19]. The University Hospital of North Norway is the only hospital serving the municipality of Tromsø, and all relevant diagnostic procedures and specialized care are exclusively performed by this hospital. The hospital discharge diagnosis registry included both outpatient clinic visits and hospitalizations. A VTE-event was recorded when all four of the following criteria were fulfilled; (i)the diagnosis was objectively verified (compression ultrasonography, venography, spiral computed tomography, perfusion-ventilation scan, pulmonary angiography or autopsy); (ii) the physician had made a diagnosis of DVT or PE in the patient's medical journal; (iii) signs and symptoms consistent with DVT or PE were present; and (iv) treatment with anticoagulants (heparin, warfarin), thrombolytic therapy or vascular surgery was required. From the autopsy registry VTEevents were identified when PE was the noted cause of death, or marked as a significant condition on the death certificate.

From the patient's medical journal, possible provoking factors other than cancer were registered. Provoking factors were: recent surgery or trauma within the previous 8 weeks,acute medical conditions (acute myocardial infarction, ischemic stroke or majorinfectious disease), marked immobilization (bedrest for $>3$ days, wheelchair use, or long-distance travelexceeding $4 \mathrm{~h}$ within the last 14 days prior to the event) orother provoking factors specifically described by a physician inthe medical record (e.g. intravascular catheter).

Study design

All subjects were included in the study during 1994-95, and followed to the date of a VTE event, migration, death, or end of the study period (Dec 31 $1^{\text {st }}$, 2009), whichever occurred first. Person-time of cancer exposure were calculated in three categories; no cancer exposure, occult cancer exposure (the one year period before cancer was diagnosed), and overt cancer exposure. Thus, subjects who developed cancer during follow-up $(n=2,290)$ contributed with non-exposed person-time from their baseline inclusion date until the date one year prior to their cancer diagnosis; then they contributed with one year of occult cancer exposure time; and overt cancer exposure time was measured from the date of manifest cancer diagnosis (Figure 1). The dataset was divided into three age groups ( $<50,50-69, \geq 70$ years), and the person-times (with and without cancer exposure) each subject spent in 
each age group were calculated. In total, the 26094 individuals contributed to 43287 observational periods. The main study outcome was incident VTE $(n=531)$, and subjects who died $(n=3,895)$ or migrated $(n=3,429)$ during follow-up were censored from the date of death or migration.

\section{Statistical analyses}

Statistical analyses were carried out using STATA version 12 (Stata corporation, College station, Texas, USA). All analyses were performed for the total cohort, as well as in the three age groups; $<50,50-69$ and $\geq 70$ years. Crude incidence rates (IRs) with corresponding 95\% confidence intervals (95\% CI) for VTE were calculatedforoccult cancer-exposure, overt cancer exposure and non-cancer exposure. Attributable risk (AR\%), the share of events among the exposed subjects that can be explained by the exposure, was calculated from incidence rates of VTE in the cancer $\left(\mathrm{I}_{\mathrm{e}}\right)$ and non-cancer $\left(\mathrm{I}_{\mathrm{o}}\right)$ population $\left(\frac{I e-I o}{I e} \cdot 100 \%\right)$. Population attributable risk fraction (PAR \%) was calculated using the incidence rates of VTE in the general population $\left(\mathrm{I}_{\mathrm{p}}\right)$ and in the non-cancer $\left(\mathrm{I}_{0}\right)$ population $\left(\mathrm{PAR} \%=\frac{I p-I o}{I p} \cdot 100 \%\right)$. AR\% and PAR\% were calculated by total cancer exposure (i.e. overt + occult cancer).

Cox proportional hazards regression models were used to calculate cause-specific hazard ratios (HR) for VTE for exposure tototal-, occult- and overt cancer.In the Cox-model, cancer was entered as a time dependent variable, age was used as timescale, and analyses were stratified by agegroups and adjusted for sex. To address potential confounding, body mass index, daily smoking (yes/no), physical activity, self-reported cardiovascular disease and diabetes at baseline were included in a multivariable model. Residual confounding by smoking quantitywas investigated by inclusion of pack-years for current smokersin an additional multivariable model. The proportional hazard assumption was verified by evaluating the parallelism between the curves of the log-log survivor function.

In order to formally test that the effect of age on risk of VTE was independent of cancer, the HR of VTE per decade increase in age adjusted for cancer was estimated by Cox-regression. 
Competing risk of death

Hypothetically, death may prevent the observation of a future potential VTE during the follow-up period, and it has been suggested that the cause specific HRs may overestimate the risk of cancer-related VTE[20]. Since the overall risk of mortality, and potentially cancer-related mortality, was expected to be different across age groups (i.e. higher in the elderly), we also calculated subhazard ratiostaking competing risk of deathinto account by using the model described by Fine and Gray[21].

\section{Results}

Baseline characteristics by age groups are shown in Table 1 . The majority of study participants were $<50$ years (63.0\%), whereas $9.5 \%$ were $\geq 70$ years at inclusion. As expected, the proportion of women was higher among the elderly. Body mass index, the proportion of subjects with diabetes and self-reported CVD increased across higher age groups, whereas the proportion of daily smokers and the frequency of both light and hard physical activity decreased (Table 1).

Among the 26,094study participants included at baseline, 2,290 (8.8\%) developed cancer during a median follow-up of 14.8 years. Cancer was more common among the elderly ( $\geq 70$ years at time of cancer diagnosis)with an incidence rate of22 per 1000 person-years, whereas the corresponding rates among the middleaged and young was 8 per 1000 and 1.8 per 1000, respectively. In the young, breast cancer was the most common cancer site in women (37\%), whereas colorectal cancer and nervous system tumours were common cancers for both sexes (Table 2).In the middle aged and elderly, prostate cancer was common in men (33\% and $26 \%$ respectively), and breast cancer was frequent in women (30\% and 13\% respectively). Colorectal- and lung cancer were frequent sites in middle-aged (14\% and $11 \%$, respectively) and elderly patients ( $19 \%$ and $16 \%$, respectively). Advanced cancer stage was more common among elderly subjects (21\%) compared to the young and middle-aged (13\% and $17 \%$, respectively) (Table 2).

A total of 531 VTE events occurredthroughout the study period (Table 3), of which 27 (5.1\%) in subjects with occult cancer, and 111 (21\%) in subjects with overt cancer (Table 4). Immobilizationwas the most common provoking factor in cancer-relatedVTE (23\%), followed by surgery (15\%), whereasamong the noncancer VTEs, surgery and immobilization were equally common (16\% and 19\%, respectively) and acute medical conditions were described in $15 \%$ of the events. The proportion of subjects with pulmonary embolism was slightly higheramong non-cancer related VTEs (39\% vs. 33\%)(Table 3). 
Overall, the crude incidence rate of VTE in the population was 1.6 per 1000person-years.Among the cancer free the incidence rate was 1.2 per 1000, whereas among the occult and overt cancer exposed the incidence rates were12.2 and 13.0per 1000 person-years, respectively (Table 4). The incidence rates of VTE increased across age groups in both exposed and unexposed subjects. In the non-cancer exposed, incidence rates of VTE in the youngest through highest age groups were0.3, 1.2 and 4.3 events per 1000 person-years, respectively. In the overt cancer population, corresponding incidence rates of VTE were9.2, 11.9and 15.1 per 1000 person-years.Among the young subjects with cancer, 96\% of the VTE events could be explained by cancer, while the corresponding proportions were $91 \%$ among middle aged and $70 \%$ among the elderly cancer patients. The overall population attributable risk (PAR\%) by cancer (overt+occult) was $24 \%$. In young subjects, $14 \%$ of the VTE events occurred due to cancer, whereas the corresponding proportions were $27 \%$ among middle-aged and $18 \%$ in the elderly (Table 4).

The overall cause specific HRs for VTE by occult cancer and overt cancer were5.2 (95\% CI: 3.5, 7.8) and 5.4 (95\% CI: 4.3, 6.7), respectively (Table 5) compared with non-cancer. The relative risk of VTE by overt cancer was highest among the young and decreased across age groups. Similar results were found for occult cancer, though we had limited power to calculate the relative risk among the young. Young subjects with overt cancer had a 26-fold increased risk of VTE (95\% CI:12.1, 56.5), whereas middle-aged and elderly had a9-fold (95\%CI: 6.6, 12.7) and 3-fold (95\% CI: 2.5, 4.5) increased risk, respectively. The hazard ratios were not altered after adjustment for body mass index, daily smoking, physical activity, self-reported cardiovascular disease and diabetes. The multivariable model adjusted forpack-years instead of daily smoking providedsimilar risk estimates (data not shown). When death was taken into account in the competing risk model, the overall risk estimate for VTE by overt cancer was attenuated from 5-fold to 3-fold. However, the subhazard ratio indicatedthat the risk was still 21-fold higherin the young, whereas the corresponding estimates for middle-aged and elderly were close to 7-fold and 2-fold, respectively.

Finally, in the total population, the risk of VTE per 10 year increment in age(HR 1.9, 95\% CI: 1.8,2.0) was only slightly attenuatedby adjustment for cancer (HR 1.8, 95\% CI: 1.7,1.9).

\section{Discussion}

We found that the overall risk of VTE in cancer patients was more than five-fold increased. Despite a higher proportion of advanced cancers in the elderly, we observed asignificant age gradient, from a26-fold increased relative risk in the youngest to a 3-fold increased relative risk in the highest age group compared to subjects 
without cancer. Crude incidence rates in the cancer and non-cancer cohorts were 13.0 and 1.2 per 1000 personyears, respectively. The attributable risk of VTE due to cancer was highest among the young and declined with increasing age from $96 \%$ in the young to $70 \%$ in the elderly. However, the proportion of VTE events that could have been saved if cancer was completely eliminatedfrom the population did not differ much inthe young and elderly(14\% vs. $18 \%$, respectively) despite thesubstantially higher (10-fold) incidence rate of cancer in the elderly. Thus, our findings suggest that the high incidence of VTE by advancing age cannot be explained by the concomitant high prevalence of cancer in the elderly.

Our finding of an overall 5-fold increased risk of VTE by cancer is inagreement withprevious cohort[16, 17] and case-control studies[4, 22]reporting a5 to 7-foldhigher risk of VTE in cancer patients. Agespecific incidence rates and relative risks were reported in a previous Danish registry study [16]that had linked a cohort of 57600 cancer patients witha cohort of 287000 controls selected from the general population.Since the primary outcome of the Danish study was hospitalization for VTE, the incidence rates and risk estimates were not directly comparable to our study which included both hospitalized and outpatient treated VTEs. Moreover, when a subgroup of the VTE diagnoses in the registry were validated, the codes for hospitalized VTE cases had a low positive predictive value (PPV $75 \%$ ), particularly when VTE was a secondary discharge diagnosis (PPV $67 \%$ [23]. However, similar to our findings, the Danish study reported increasing incidence rates of VTE across older age groups among cancer patients, while the relative risks of VTE by cancer was highest among the young. A recent linkage of UK databases[17] with 83000 cancer patients and 580000 controls reported a HR of 4.7 for all cancers combined, and higher IR in subjects $>60$ years, compared to those $<60$ years. For certain high risk sites (e.g. pancreas, lung, mesothelioma), the IRs were higher in young subjects.

Similar to our findings the UK linked databases found an overall IR of 14 per 1000 person-years, and a meta-analysis of 38 papers on cohorts with cancer patients (of which 7 were of average risk patients) reported an IR of 13 per 1000 person-years[24].

VTE is a multicausal disease[25] among which age is one of the most consistent and strongest risk factors. Along with an age-dependent increase in the incidence of cancer, a substantial increase in PAR of cancer for VTE by age would be expected. Previously, few attempts have been made to estimate the PAR of cancer for VTE in the young and elderly [15, 26].Engbers and colleagues estimated the PAR to be $15 \%$ in the younger population and 35\% in the elderly, based on an assumption that the relative risk of VTE was constant across age groups in cancer patients [26]. In our study, we were able to calculate PAR based on actual incidence rates of cancer and VTE using a general population cohort followed closely for a maximum of 14 years. As previous 
studies have indicated $[15,16]$, our data confirmed that on a relative scale cancer was associated with significantly lower risk of VTE in the elderly than in the young. Thus, the proportion of events that could be attributed to cancerincreased only slightly by increasing age.In coherence with these findings, the HR for VTE per 10 year increment in age remained virtually similar after adjustment for cancer in the total population.

The absolute risk difference between cancer and non-cancer was similar in the three age groups and ranged from 7 to 11 cases per 1000 persons per year. Since cancer is substantially more common in elderly subjects, it could be reasonable to assume that cancer would lead to a higher proportion of VTE events among the elderly at a population level. However, in our study cancer was responsible for $27 \%$ of the VTE cases among middle aged subjects, and only $18 \%$ of the VTEs in patients aged 70 years or older. Thus, clearly, cancer is not the main contributor to the increased incidence of VTE by advancing age. A number of comorbidities and VTE risk factors become more frequent with increasing age. Ischemic heart disease, stroke, severe infections and hospitalizations all accumulate in the elderly. Other risk factors like age-related changes in vessel walls and valves, or distributed flow due to less muscle strength and tone, may also contribute to increased VTE risk. Further on, the share of VTE events with inherited thrombophilia decline with increasing age[27], suggesting that acquired risk factors becomegradually moreimportantat higher ages. Consequently, presence of other risk factors for VTE may dilute the relative importance of cancer as a risk factor in older patients. In a previous study[26], the attributable risk for VTE in cancer patients was estimated to be $86 \%$ in both young and elderly patients with cancer. However, we observed a gradient in attributable risk from $96 \%$ in young cancer patients to $70 \%$ among elderly cancer patients. This observation supports the notion that accumulated co-morbidities and other VTE risk factors diluted the impact of cancer on VTE risk in the elderly. From another perspective, the risk associated with cancer may be more pronounced in young and middle-aged subjects due to a more adversecourse of the malignant disease and/or more aggressive cancer therapy in these patients.

With regard to aetiology, the cause specific HRs are the most important in assessment of cancer-related VTE risk. However, it has been claimed that mortality should be considered a competing risk factor when studying the occurrence of VTE events in a cancer population [20], since the cause specific HRs may overestimate the risk of cancer-related VTE. When competing risk by death was taken into account in our study, the risk estimates of VTE by overt cancer were attenuated in all age groups, and as expected, most attenuated among the elderly due to higher mortality rate in this age group.

A clear advantage of our study is the high completeness and validity of the VTE events. Furthermore, the high participation rate among people recruited from a general population, and the prospective study design 
with long follow-up time contribute to a high quality. As cancer is a relatively rare exposure in the general population, a limitation of our study is the size of the exposed population, which prohibits the use of smaller age ranges in the calculations and sub-group analysis of specific cancer sites. Since cancer is already a wellestablished risk factor for VTE, diagnostic bias may be presentdue to a lower threshold for performing the diagnostic proceduresof VTE in subjects with manifest malignancy. Moreover, some of the apparently cancer free subjects who died during follow-up may have had occult cancer. Thus, fatal venous thrombotic events in patients with occult cancercould be misclassified as non-cancer exposed events, and therefore our risk estimates by occult cancer may be slightly underestimated.Finally, information about cancer treatmentwas not available in our study. Since the frequency of different treatment modalitiesmayvary according to age, treatment represents a potential confounder in the comparison of the age-groups that could not be taken into account.

In conclusion, the relative risk of VTE by cancer was higher in young subjects, and the population attributable risk declined considerably from middle-aged to elderly subjects. Thus, our findings suggest that cancer is not a major contributor to the increased risk of VTE by advancing age, and that VTE would continue to be a disease substantially more common in the elderly if cancer did not exist in the population.

\section{Acknowledgments}

The authors received grants from the Northern Norway Regional Health Authority (SKBrækkan and JB Hansen) and the Tom Wilhelmsen Foundation (K Blix).KB, SKB and JBH were involved in acquisition of the data, concept and design of the study, data analysis, interpretation of results and drafting the manuscript. SleC was involved in data analysis, interpretation and critical revision of the manuscript. FES and SCC contributed to interpretation of the results and critical revision of the manuscript. All authors approved the final version of the manuscript.

\section{Conflict of interests}

The authors declare that they have no conflict of interest. 


\section{References}

1. Khorana AA, Francis CW, Culakova E, Kuderer NM, Lyman GH. Thromboembolism is a leading cause of death in cancer patients receiving outpatient chemotherapy. $\mathrm{J}$ Thromb Haemost. 2007;5(3):632-4. doi:10.1111/j.1538-7836.2007.02374.x

2. Naess IA, Christiansen SC, Romundstad P, Cannegieter SC, Rosendaal FR, Hammerstrom J. Incidence and mortality of venous thrombosis: a population-based study. J Thromb Haemost. 2007;5(4):692-9. doi:10.1111/j.1538-7836.2007.02450.x

3. Noble S, Pasi J. Epidemiology and pathophysiology of cancer-associated thrombosis. Br J Cancer. 2010;102 Suppl 1:S2-9. doi:10.1038/sj.bjc.6605599

4. Blom JW, Doggen CJ, Osanto S, Rosendaal FR. Malignancies, prothrombotic mutations, and the risk of venous thrombosis. Jama. 2005;293(6):715-22. doi:10.1001/jama.293.6.715

5. Cushman M, Tsai AW, White RH, et al. Deep vein thrombosis and pulmonary embolism in two cohorts: the longitudinal investigation of thromboembolism etiology. Am J Med. 2004;117(1):19-25. doi:10.1016/j.amjmed.2004.01.018

6. Braekkan SK, Borch KH, Mathiesen EB, Njolstad I, Wilsgaard T, Hansen JB. Body height and risk of venous thromboembolism: The Tromso Study. Am J Epidemiol. 2010;171(10):1109-15. doi:10.1093/aje/kwq066

7. Piccioli A, Falanga A, Baccaglini U, Marchetti M, Prandoni P. Cancer and venous thromboembolism. Semin Thromb Hemost. 2006;32(7):694-9. doi:10.1055/s-2006-951297

8. $\quad$ Elting LS, Escalante CP, Cooksley C, et al. Outcomes and cost of deep venous thrombosis among patients with cancer. Arch Intern Med. 2004;164(15):1653-61. doi:10.1001/archinte.164.15.1653

9. Prandoni P, Lensing AW, Piccioli A, et al. Recurrent venous thromboembolism and bleeding complications during anticoagulant treatment in patients with cancer and venous thrombosis. Blood. 2002;100(10):3484-8. doi:10.1182/blood-2002-01-0108

10. Levitan N, Dowlati A, Remick SC, et al. Rates of initial and recurrent thromboembolic disease among patients with malignancy versus those without malignancy. Risk analysis using Medicare claims data. Medicine. 1999;78(5):285-91.

11. Sorensen HT, Mellemkjaer L, Olsen JH, Baron JA. Prognosis of cancers associated with venous thromboembolism. N Engl J Med. 2000;343(25):1846-50.

doi:10.1056/NEJM200012213432504

12. Chew HK, Wun T, Harvey D, Zhou H, White RH. Incidence of venous thromboembolism and its effect on survival among patients with common cancers. Arch Intern Med. 2006;166(4):458-64. doi:10.1001/.458

13. Braekkan SK, Mathiesen EB, Njolstad I, Wilsgaard T, Stormer J, Hansen JB. Family history of myocardial infarction is an independent risk factor for venous thromboembolism: the Tromso study. J Thromb Haemost. 2008;6(11):1851-7. doi:10.1111/j.15387836.2008.03102.x

14. Norway S. Statistical yearbook of Norway 2009. 2009.

15. Rosendaal FR, A VANHV, Doggen CJ. Venous thrombosis in the elderly. J Thromb Haemost. 2007;5 Suppl 1:310-7. doi:10.1111/j.1538-7836.2007.02489.x

16. Cronin-Fenton DP, Sondergaard F, Pedersen LA, et al. Hospitalisation for venous thromboembolism in cancer patients and the general population: a population-based cohort study in Denmark, 1997-2006. Br J Cancer. 2010;103(7):947-53. doi:10.1038/sj.bjc.6605883 17. Walker AJ, Card TR, West J, Crooks C, Grainge MJ. Incidence of venous thromboembolism in patients with cancer - a cohort study using linked United Kingdom databases. Eur J Cancer. 2013;49(6):1404-13. doi:10.1016/j.ejca.2012.10.021 
18. Larsen IK, Smastuen M, Johannesen TB, et al. Data quality at the Cancer Registry of Norway: an overview of comparability, completeness, validity and timeliness. Eur J Cancer. 2009;45(7):1218-31. doi:10.1016/j.ejca.2008.10.037

19. Braekkan SK, Mathiesen EB, Njolstad I, Wilsgaard T, Stormer J, Hansen JB. Mean platelet volume is a risk factor for venous thromboembolism: the Tromso Study, Tromso, Norway. J Thromb Haemost. 2010;8(1):157-62. doi:10.1111/j.1538-7836.2009.03498.x 20. Campigotto F, Neuberg D, Zwicker JI. Accounting for death as a competing risk in cancer-associated thrombosis studies. Thromb Res. 2012;129 Suppl 1:S85-7. doi:10.1016/S0049-3848(12)70023-3

21. Fine J, Gray R. A proportional hazards model for the subdistribution of a competing risk Journal of the american statistical association. 1999(94):496-509.

22. Heit JA, Silverstein MD, Mohr DN, Petterson TM, O'Fallon WM, Melton LJ, 3rd. Risk factors for deep vein thrombosis and pulmonary embolism: a population-based casecontrol study. Arch Intern Med. 2000;160(6):809-15.

23. Severinsen MT, Kristensen SR, Overvad K, Dethlefsen C, Tjonneland A, Johnsen SP. Venous thromboembolism discharge diagnoses in the Danish National Patient Registry should be used with caution. Journal of clinical epidemiology. 2010;63(2):223-8.

doi:10.1016/j.jclinepi.2009.03.018

24. Horsted F, West J, Grainge MJ. Risk of venous thromboembolism in patients with cancer: a systematic review and meta-analysis. PLoS Med. 2012;9(7):e1001275.

doi:10.1371/journal.pmed.1001275

25. Rosendaal FR. Venous thrombosis: a multicausal disease. Lancet. 1999;353(9159):1167-73.

26. Engbers MJ, van Hylckama Vlieg A, Rosendaal FR. Venous thrombosis in the elderly: incidence, risk factors and risk groups. J Thromb Haemost. 2010;8(10):2105-12.

doi:10.1111/j.1538-7836.2010.03986.x

27. Weingarz L, Schwonberg J, Schindewolf M, et al. Prevalence of thrombophilia according to age at the first manifestation of venous thromboembolism: results from the MAISTHRO registry. Br J Haematol. 2013;163(5):655-65. doi:10.1111/bjh.12575 
Table 1 Baseline characteristics according to age groups at inclusion in the Tromsø IV Study (1994-95).

\begin{tabular}{lccc}
\hline & \multicolumn{3}{c}{ Age groups (years) } \\
\cline { 2 - 3 } & $<50$ & $\mathbf{5 0 - 6 9}$ & $\geq \mathbf{7 0}$ \\
\hline Distribution & $63.0(16442)$ & $27.5(7171)$ & $9.5(2481)$ \\
Age (years) & $37.5 \pm 7.1$ & $58.7 \pm 5.9$ & $76.4 \pm 4.8$ \\
Sex (female) & $52.2(8589)$ & $50.2(3598)$ & $59.1(1467)$ \\
BMI (kg/m $\left.{ }^{2}\right)$ & $24.6 \pm 3.6$ & $26.1 \pm 3.9$ & $26.2 \pm 4.3$ \\
Daily smoking & $40.5(6$ 651) & $35.7(2554)$ & $20.1(496)$ \\
Physical activity* & $37.3(6104)$ & $22.7(1614)$ & $9.4(231)$ \\
Self-reported diabetes & $0.6(100)$ & $2.4(175)$ & $7.2(177)$ \\
Self-reported CVD & $1.0(169)$ & $10.3(736)$ & $29.8(738)$
\end{tabular}

Values are mean \pm 1 standard deviation or percentages with numbers in parentheses.*More than one hour per week. $\mathrm{BMI}=$ Body mass index, $\mathrm{CVD}=$ Cardiovascular disease 
Table 2 Distribution of cancer sites and stage at diagnosis by age groups. The Tromsø Study 1994-2009.

\begin{tabular}{|c|c|c|c|c|}
\hline & \multicolumn{3}{|c|}{ Age groups (years) } & \multirow[b]{2}{*}{ Total } \\
\hline & $<50$ & $50-69$ & $\geq 70$ & \\
\hline & $\mathrm{n}=261$ & $\mathrm{n}=1053$ & $\mathrm{n}=976$ & $\mathrm{n}=2290$ \\
\hline & $\%$ & $\%$ & $\%$ & $\%$ \\
\hline \multicolumn{5}{|l|}{ Cancer site } \\
\hline Colon/rectum & 8.8 & 13.7 & 18.8 & 15.3 \\
\hline Prostate & 5.0 & 33.0 & 26.1 & 27.9 \\
\hline Lung & 5.0 & 11.1 & 15.9 & 12.5 \\
\hline Breast & 37.0 & 29.9 & 13.2 & 24.5 \\
\hline Gynecological & 19.3 & 13.7 & 12.3 & 14.0 \\
\hline Upper GI* & 3.5 & 4.3 & 6.5 & 5.1 \\
\hline Bladder and urinary tracts & 1.5 & 4.8 & 5.8 & 4.9 \\
\hline Hematopoietic system & 3.8 & 4.0 & 4.8 & 4.3 \\
\hline CNS/PNS & 8.1 & 4.8 & 2.0 & 3.9 \\
\hline Lymphatic system & 3.5 & 3.4 & 3.6 & 3.5 \\
\hline Pancreas & 1.5 & 2.2 & 4.7 & 3.2 \\
\hline All other sites & 23.8 & 13.8 & 12.2 & 14.2 \\
\hline \multicolumn{5}{|l|}{ Cancer stage } \\
\hline Localized disease & 44.1 & 35.2 & 30.1 & 32.1 \\
\hline Regional disease & 21.1 & 21.7 & 21.4 & 21.5 \\
\hline Distant metastasis & 12.6 & 16.9 & 20.7 & 18.0 \\
\hline Unknown stage & 22.2 & 26.2 & 27.8 & 26.4 \\
\hline
\end{tabular}

For breast, prostate and gynecological cancers sex-specific percentages are presented. * Esophagus, stomach and small intestine. 
Table 3Other provoking factors for venous thromboembolism (VTE) in cancer and non-cancer patients at the time of VTE diagnosis. The Tromsø Study 1994-2009.

\begin{tabular}{|c|c|c|c|c|}
\hline & \multicolumn{4}{|c|}{ Age (years) } \\
\hline & $<50$ & $50-69$ & $\geq 70$ & Total \\
\hline Cancer related VTE (n) & 8 & 65 & 65 & 138 \\
\hline Surgery* & $12.5(1)$ & $20(13)$ & $9.2(6)$ & $14.5(20)$ \\
\hline Acute medical condition $^{\dagger}$ & 0 & $9.2(6)$ & $21.5(14)$ & $14.5(20)$ \\
\hline Trauma* & 0 & 0 & $1.5(1)$ & $0.7(1)$ \\
\hline Immobilization $^{\ddagger}$ & $37.5(3)$ & $20(13)$ & $24.6(16)$ & $23.2(32)$ \\
\hline Other provoking factor ${ }^{\S}$ & $25.0(2)$ & $7.7(5)$ & $4.6(3)$ & $7.2(10)$ \\
\hline Total provoked" & $0.63(5)$ & $46.2(30)$ & $44.6(29)$ & $46.4(64)$ \\
\hline Deep vein thrombosis & $75.0(6)$ & $69.2(45)$ & $58.5(38)$ & $64.5(89)$ \\
\hline Pulmonary embolism & $25.0(2)$ & $30.8(20)$ & $41.5(27)$ & $35.5(49)$ \\
\hline Non-cancer related VTE(n) & 48 & 151 & 194 & 393 \\
\hline Surgery* & $10.4(5)$ & $17.2(26)$ & $16.5(32)$ & $16.0(63)$ \\
\hline Acute medical condition $^{\dagger}$ & $10.4(5)$ & $9.9(15)$ & $19.6(38)$ & $14.8(58)$ \\
\hline Trauma* & $14.6(7)$ & $8.6(13)$ & 6.7 (13) & $8.4(33)$ \\
\hline Immobilization $^{\ddagger}$ & $22.9(11)$ & $18.5(28)$ & $18.6(36)$ & $19.1(75)$ \\
\hline Other provoking factor ${ }^{\S}$ & $0.21(1)$ & $4.6(7)$ & $2.6(5)$ & $3.3(13)$ \\
\hline Total provoked" & $41.7(20)$ & 45.7 (69) & 45.9 (89) & $45.3(178)$ \\
\hline Deep vein thrombosis & $62.5(30)$ & $58.9(89)$ & $58.2(113)$ & $59.0(232)$ \\
\hline Pulmonary embolism & $37.5(18)$ & $41.1(62)$ & $41.8(81)$ & $41.0(161)$ \\
\hline
\end{tabular}

Values are numbers or percentages with numbers in parentheses. *Within 8 weeks before the VTE event. ${ }^{\dagger}$ Myocardial infarction, ischemic stroke or major infectious disease. ${ }^{\ddagger}$ Bed rest $>3$ days, wheelchair, long haul travel $>4$ hours in the past 14 days. ${ }^{\S}$ Other provoking factor described by the physician, e.g. intravascular catheter. "One or more provoking factors. 
Table 4 Incidence rates (IR) with 95\% confidence intervals (CI) per 1000 person-years, attributable risk fraction(AR \%) and population attributable risk fraction (PAR \%) of venous thromboembolism (VTE) by overt and occult cancer respectively. The Tromsø Study 1994-2009.

\begin{tabular}{|c|c|c|c|c|}
\hline & $\begin{array}{c}\text { Total } \\
\text { All age groups }\end{array}$ & $<50$ years & 50-69 years & $\geq 70$ years \\
\hline \multicolumn{5}{|l|}{ Occult cancer } \\
\hline Person years & 2213 & 270 & 1034 & 909 \\
\hline Events & 27 & 0 & 16 & 11 \\
\hline IR (95\% CI) & $12.2(8.4-17.8)$ & 0 & $15.5(9.5-25.5)$ & $12.1(6.7-21.9)$ \\
\hline \multicolumn{5}{|l|}{$\begin{array}{l}\text { Overt cancer } \\
\text { overall }\end{array}$} \\
\hline Person years & 8550 & 874 & 4108 & 3568 \\
\hline Events & 111 & 8 & 49 & 54 \\
\hline IR (95\% CI) & $13.0(10.8-15.6)$ & $9.2(4.6-18.3)$ & $11.9(9.0-15.8)$ & $15.1(11.6-19.8)$ \\
\hline \multicolumn{5}{|l|}{ No cancer } \\
\hline Person years & 318080 & 147901 & 126021 & 44158 \\
\hline Events & 393 & 48 & 151 & 194 \\
\hline IR (95\% CI) & $1.2(1.1-1.4)$ & $0.3(0.2-0.4)$ & $1.2(1.0-1.4)$ & $4.4(3.8-5.1)$ \\
\hline \multicolumn{5}{|c|}{ Total cancer (overt+occult) } \\
\hline Person years & 10763 & 1144 & 5142 & 4477 \\
\hline Events & 138 & 8 & 65 & 65 \\
\hline IR (95\% CI) & $12.8(10.9-15.2)$ & $7.0(3.5-14.0)$ & $12.6(9.9-16.1)$ & $14.5(11.4-18.5)$ \\
\hline \multicolumn{5}{|c|}{ Attributable risks (overt+occult) } \\
\hline AR \% (95\% CI) & 90.6 & 95.7 & 90.5 & 70.3 \\
\hline PAR \% (95\% CI) & 23.5 & 13.6 & 27.2 & 17.5 \\
\hline
\end{tabular}


Table 5 Hazard ratios (HR) of venous thromboembolism (VTE) by overt and occult cancer, respectively. The Tromsø Study 1994-2009.

\begin{tabular}{|c|c|c|c|c|}
\hline & \multicolumn{4}{|c|}{ Age-groups } \\
\hline & All ages & $<50$ & $50-69$ & $\geq 70$ \\
\hline \multicolumn{5}{|l|}{ Occult cancer } \\
\hline Cause specific HR $(95 \% \mathrm{CI})^{\mathrm{a}}$ & $5.2(3.5-7.8)$ & - & $11.6(6.9-19.4)$ & $2.8(1.5-5.1)$ \\
\hline Cause specific HR (95 \% CI) ${ }^{\mathrm{b}}$ & $5.1(3.4-7.7)$ & - & $11.5(6.9-19.4)$ & $2.5(1.3-4.8)$ \\
\hline \multicolumn{5}{|l|}{ Overt cancer } \\
\hline Cause specific HR $(95 \% \text { CI })^{\mathrm{a}}$ & $5.4(4.3-6.7)$ & $26.1(12.1-56.5)$ & $9.2(6.6-12.7)$ & $3.4(2.5-4.5)$ \\
\hline Cause specific HR (95 \% CI) & $5.5(4.4-6.8)$ & $27.6(12.6-60.5)$ & $9.2(6.7-12.8)$ & $3.4(2.5-4.6)$ \\
\hline Competing risk HR $(95 \% \mathrm{CI})^{\mathrm{a}}$ & $2.8(2.2-3.6)$ & $21.7(9.5-49.7)$ & $6.6(4.7-9.4)$ & $1.8(1.3-2.5)$ \\
\hline Competing risk HR $(95 \% \mathrm{CI})^{\mathrm{b}}$ & $2.9(2.3-3.8)$ & $22.9(9.8-53.7)$ & $6.8(4.8-9.6)$ & $1.8(1.3-2.5)$ \\
\hline \multicolumn{5}{|l|}{ Total cancer (occult+overt) } \\
\hline Cause specific HR $\left(95 \%\right.$ CI) ${ }^{\mathrm{a}}$ & $5.3(4.4-6.5)$ & $19.9(9.2-43.0)$ & $9.6(7.2-13.0)$ & $3.2(2.4-4.3)$ \\
\hline Cause specific HR $\left(95 \%\right.$ CI) ${ }^{b}$ & $5.4(4.4-6.6)$ & $20.5(9.4-44.7)$ & $9.7(7.2-13.1)$ & $3.2(2.4-4.3)$ \\
\hline
\end{tabular}

a Adjusted for sex.

${ }^{\mathrm{b}}$ Adjusted for sex, body mass index, smoking status, physical activity, self-reported cardiovascular disease and diabetes. 
Figure 1Definition of exposure time

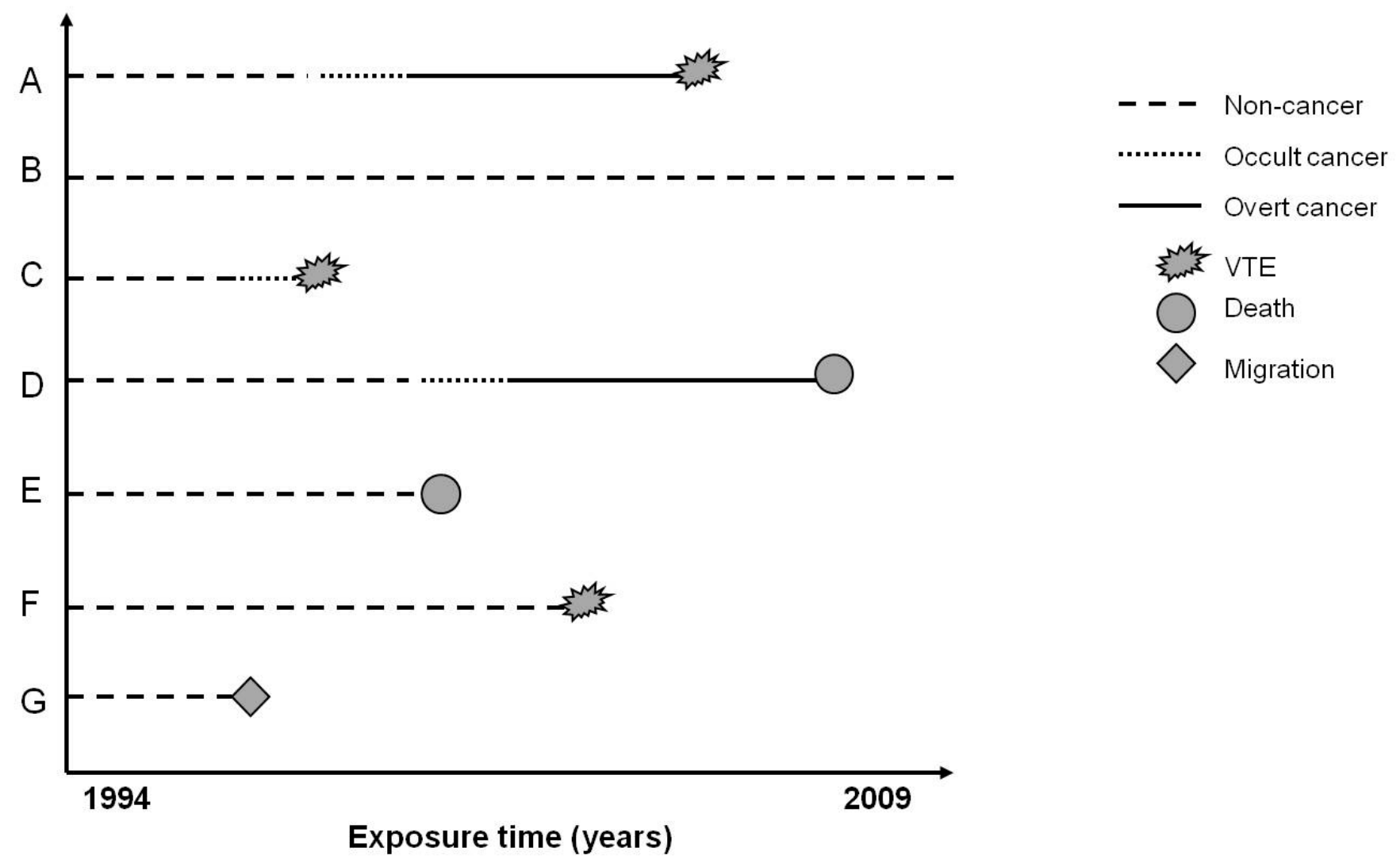

Abbreviations: VTE, venous thromboembolism.

Examples of exposure time measured in patients during follow-up. Person A: contributed with non-exposed person-time from inclusion until 1-year prior to cancer diagnosis; then 1-year occult cancer time followed, and overt cancer exposure was calculated from diagnosis until VTE event. Person B: non-cancer exposed through the whole study period. Person C: occult cancer related VTE. 